\title{
Overview of strong interaction from kaonic atoms
}

\author{
E. Friedman ${ }^{1, *}$ \\ ${ }^{1}$ Racah Institute of Physics, the Hebrew University, Jerusalem, Israel
}

\begin{abstract}
Experiments on kaonic atoms between 1972 and 1982 at four worldclass laboratories established a data base comprising of 24 species along the periodic table. Here we present a short overview of methods for obtaining strong interaction information from these results. Early straight-forward phenomenological analyses and also more sophisticated approaches led to ambiguities and open problems. Using state-of-the-art $\bar{K}$-nucleon amplitudes plus a phenomelogical multinucleon term can fit the data only when sub-threshold energies are considered. Remaining ambiguities are removed with the help of old bubblechamber experiments on single-nucleon absorption fraction at threshold.
\end{abstract}

\section{Introduction}

Less than a decade after the first observation of X-ray cascades from kaonic atoms, strong interaction level shifts and widths were measured directly, in addition to experimental transition yields that indirectly provide information on level widths. Experiments between 1972 and 1982 provided good quality data for 24 nuclear species from Li to $\mathrm{U}$, that serve up to now as the data-base for studies of low energy antikaon interaction with nuclei. Puzzles with early results for kaonic atoms of hydrogen and He were resolved by new precision experiments at KEK and Frascati between 1997 and 2007. These are excluded from the present overview. Early phenomenological analyses based on the optical model indicated departure of potentials from the geometry of charge distribution of nuclei, thus suggesting the use of density-dependent (DD) phenomenological interaction models. Good fits to the data were achieved but conflicting results were obtained from attempts to relate experiments to more fundamental antikaon-nucleon interaction.

In Sect. 2 we outline the results of simple as well as DD phenomenological potentials and in Sect. 3.1 we discuss examples of potentials based on antikaon-nucleon interaction models with amplitudes taken at threshold. The need to properly average results over subthreshold energies is discussed and in Sect. 3.2 a sub-threshold kinematical model is outlined. This model is applied to seven state-of-the-art $\bar{K}$-nucleon amplitudes and agreement with experiment is reached only when these are supplemented by a phenomenological DD term, representing multinucleon processes. However, ambiguities between the models still remain. In Sect. 4 the ambiguities are removed when old bubble-chamber experiments on singlenucleon absorption fraction at threshold are considered. Sect. 5 is a summary.

\footnotetext{
*e-mail: elifried@cc.huji.ac.il
} 


\section{Phenomenological analyses}

In seeking strong-interaction information from kaonic atom experiments we direct our attention to 'global' fits to experimental results, handling together nuclear species over the whole of the periodic table. This approach is aimed at providing an average behaviour of the $\bar{K}$ nucleus system. Obviously local variations cannot be identified and could affect the resulting picture. The simplest optical potential $V_{K^{-}}$is the generic $t \rho(r)$ potential, which for $s$-wave $K^{-}$-nucleon interactions is:

$$
2 \mu V_{K^{-}}(r)=-4 \pi\left(1+\frac{A-1}{A} \frac{\mu}{M}\right)\left\{b_{0}\left[\rho_{n}(r)+\rho_{p}(r)\right]+b_{1}\left[\rho_{n}(r)-\rho_{p}(r)\right]\right\} .
$$

$\rho_{n}$ and $\rho_{p}$ are the neutron and proton density distributions, $M$ is the mass of the nucleon, $\mu$ is the reduced mass. From experience the parameter $b_{1}$ is poorly determined and we ignore it in what follows. Good fits $\left(\chi^{2}=129\right.$ for 65 points $)$ lead to $b_{0}=0.63 \pm 0.06+\mathrm{i}(0.89 \pm 0.05) \mathrm{fm}$, which in the impulse approximation is minus the scattering amplitude at threshold. From phase-shifts one gets $b_{0}=-0.15+\mathrm{i} 0.63 \mathrm{fm}$ meaning that the low-density limit is not respected. Early analyses [1] suggested that the geometry of the optical potential is different from that of the nuclear charge distribution, indicating that $b_{0}$ of Eq. (1) may depend on density.

To respect the low density limit $b_{0}$ was replaced by $-0.15+\mathrm{i} 0.63+B_{0}\left[\rho(r) / \rho_{0}\right]^{\alpha}$, with $\rho_{0}$ the central nuclear density. Varying the complex parameter $B_{0}$ and $\alpha, \chi^{2}$ could be lowered by as much as 25 units [2]. (This reference contains a full listing of the data.) The real and imaginary parts of the potential indeed had geometries different from the charge distribution. The real potential at the nuclear center went up from typically $75 \mathrm{MeV}$ to about $180 \mathrm{MeV}$ whereas the imaginary potential went down from $120 \mathrm{MeV}$ to $80 \mathrm{MeV}$. The deep real potential caused some interest at the time because if extrapolated to neutron star densities it could allow the presence of protons and $K^{-}$mesons in thermal equilibrium with neutrons, with consequences to the equation of state (and mass) of neutron stars. At present this seems not to be an attractive option. Deep potentials could support strongly bound $K^{-}$nuclear states, although their huge widths will make them unobservable. The ability to determine the potential close to the nuclear center is discussed in Sect. 4.

\section{Single-nucleon based models}

\subsection{Amplitudes at threshold}

Attempts to go beyond purely phenomenological approaches were made in 2000 by Ramos and Oset [3] and tested by Baca et al. [4] and independently by Cieplý et al. [5]. In both models the $\bar{K}$-nucleon amplitudes were constructed from a chiral approach, respecting several experimental cross sections at very low energies and three reaction branching ratios at threshold. In both cases the optical potentials led to poor agreement with kaonic atom experiments and it was possible to reach agreement with experiment barely comparable with that achieved with phenomenological potentials only by adjusting some of the parameters by up to $50 \%$.

Another approach was to add to the $\bar{K}$-nucleon amplitude a phenomenological term and to fit the latter to the data. Reasonably good fits could be obtained but a common feature to all the solutions was that the added term had a negative imaginary part. Expecting that to a valid single-nucleon based amplitude an added term would represent true absorption by multinucleon processes, the negative imaginary term indicated that there was a basic fault in the whole approach.

The proximity of the $\Lambda(1405)$ resonance to the $\bar{K}$-nucleon threshold had been known to cause rapid variations in the respective amplitudes near threshold. Indeed as early as in 1971 
Wycech [6] and Bardeen et al. [7] suggested that subthreshold energies were relevant for the $K^{-}$nucleus interaction in kaonic atoms. However, limited data base prevented full evaluation of this idea although Bardeen et al. showed its success in a few examples.

\subsection{Sub-threshold kinematics model}

The strong interaction shift and width of the $1 s$ state in kaonic atoms of hydrogen have been available with good precision for several years now due to the SIDDHARTA collaboration [8, 9] and these data have been used as constraints in recent models for the $\bar{K}$-nucleon interaction, see [10] for discussion and notations.
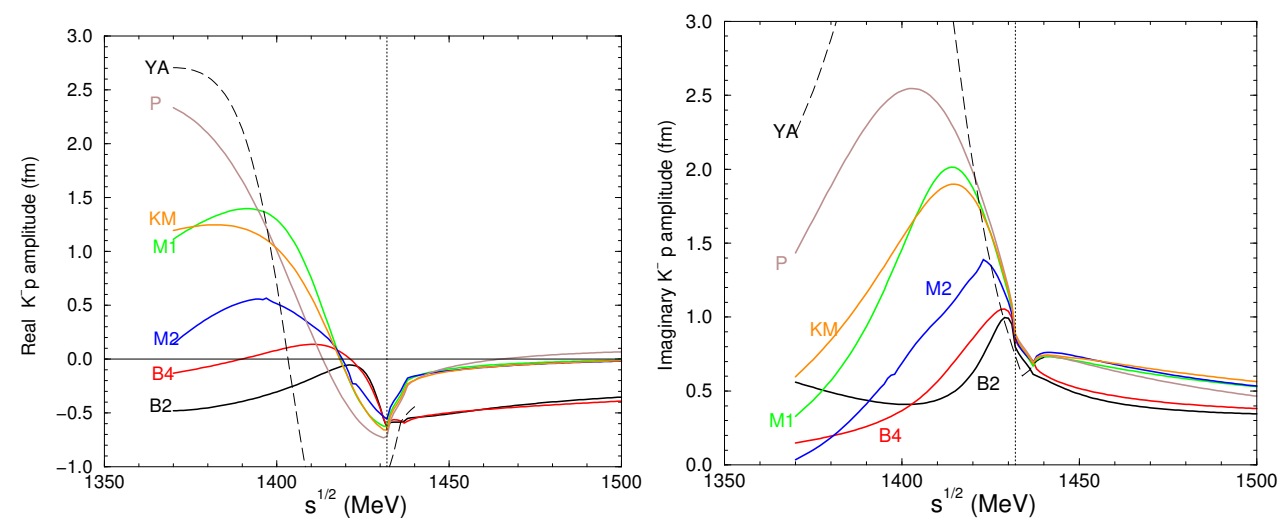

Figure 1. $K^{-} p s$-wave amplitudes used in the present work, taken from chiral SU(3) meson-baryon coupled-channel dynamical models discussed in [10] and denoted there by KM for Kyoto-Munich [11], $\mathrm{P}$ for Prague [12], M1 and M2 for Murcia [13], and B1 and B2 for Bonn [14]. The YA curves (kindly supplied by T. Hyodo) are for a fixed energy-independent potential of [15]. Vertical dotted lines indicate threshold energies.

Fig. 1 shows predicted $K^{-} p$ amplitudes from six recent chiral models and the corresponding amplitude for the earlier YA model [15] based on energy-independent potential. The rapid variation of the amplitudes near threshold is self evident and we turn now to the question of how to choose the relevant energy of the $K^{-} N$ amplitudes when constructing an optical potential.

Choosing the Mandelstam variable $s=\left(E_{K^{-}}+E_{N}\right)^{2}-\left(\vec{p}_{K^{-}}+\vec{p}_{N}\right)^{2}$ as the argument for specifying the amplitude in a kaonic atom, we first note that the relevant energy is below threshold, at least by a non-negligible nucleon binding energy and by a smaller $K^{-}$binding energy in the atom. In addition we note that in the kaonic atom system $\vec{p}_{K^{-}}+\vec{p}_{N}$ does not vanish, unlike in the free $K^{-} N$ system. When addressing only averages then it is easy to see that the average of $\left(\vec{p}_{K^{-}}+\vec{p}_{N}\right)^{2}$ is given by the average of $\vec{p}_{N}^{2}+\frac{A-2}{A} \vec{p}_{K^{-}}^{2}$ thus reducing further the relevant energy. In practice [16-19] $\vec{p}_{N}^{2}$ is taken from the Fermi gas model at the local density and $\vec{p}_{K^{-}}^{2}$ is taken from the $K^{-}$energy and the local potential. Since the energy at which the amplitude is evaluated depends on the potential and the potential depends on the amplitude, a self-consistent solution is required at each point. Between 4 and 6 iterations usually produce a well-defined density-dependent optical potential. Finally, corrections due to Pauli correlations [20] are also included.

Table 1 shows results when using the seven amplitudes within the sub-threshold kinematics approach, without any adjustments and it is obvious that there is no agreement with 
Table 1. $\chi^{2}$ for 65 kaonic atoms data points from potentials based on single-nucleon amplitudes.

\begin{tabular}{lllllll|l}
\hline model & B2 & B4 & M1 & M2 & P & KM & YA \\
\hline$\chi^{2}(65)$ & 1174 & 2358 & 2544 & 3548 & 2300 & 1806 & 2116 \\
\hline
\end{tabular}

experiment. As a next step a phenomenological term of $B\left(\rho / \rho_{0}\right)^{\alpha}$ is added to the above singlenucleon based amplitudes, adjusting the complex parameter $B$ and the exponent $\alpha$ [19].

Table 2. Fits to 65 kaonic atoms data points when single-nucleon amplitudes are supplemented by a $B\left(\rho / \rho_{0}\right)^{\alpha}$ term with fixed $\alpha$ compatible with its best-fit value. $B$ in units of fm.

\begin{tabular}{lllllll|l}
\hline model & B2 & B4 & M1 & M2 & P & KM & YA \\
\hline$\alpha$ & 0.3 & 0.3 & 0.3 & 1.0 & 1.0 & 1.0 & 1.0 \\
$\operatorname{Re} B$ & $2.4 \pm 0.2$ & $3.1 \pm 0.1$ & $0.3 \pm 0.1$ & $2.1 \pm 0.2$ & $-1.3 \pm 0.2$ & $-0.9 \pm 0.2$ & $-2.0 \pm 0.2$ \\
$\operatorname{Im} B$ & $0.8 \pm 0.1$ & $0.8 \pm 0.1$ & $0.8 \pm 0.1$ & $1.2 \pm 0.2$ & $1.5 \pm 0.2$ & $1.4 \pm 0.2$ & $0.65 \pm 0.2$ \\
$\chi^{2}(65)$ & 111 & 105 & 121 & 109 & 125 & 123 & 150 \\
\hline
\end{tabular}

Table 2 shows the best fits obtained with the additional term. Because of strong correlations between $B$ and the exponent $\alpha$ we show results when gridding on the latter. For the six chiral amplitudes the fits are good and, moreover, the imaginary part of $B$ is positive and very well defined, thus representing absorption in addition to that due to the interaction of $K^{-}$ with single nucleons. This is in sharp contrast with early attempts to use chiral amplitudes at threshold as discussed in Sect. 3.1. We note also that without the Pauli correlations the imaginary part of $B$ turns out negative. With the present sub-threshold algorithm all six chiral amplitudes with their respective added phenomenological term are acceptable as representing $K^{-}$-nucleus interaction in kaonic atoms. The six models are essentially equivalent at this point and one needs additional information in order to choose a preferred one, if any.

\section{Single-nucleon absorption fractions}

Useful additional information is the fraction of multinucleon absorptions at rest, determined long ago by bubble chamber experiments [21-23]. Distinction between single and multinucleon processes is possible because absorptions on a single nucleon are characterized by emission of pions, $K^{-}+N \rightarrow Y+\pi$, whereas multinucleon absorptions are pionless $K^{-}+N+N \rightarrow Y+N$. Averaging results from the above three experiments one finds $25 \pm 5 \%$ as the fraction of multinucleon absorptions at rest for nuclei heavier than carbon, with no evidence for a significant dependence on the charge for heavier species.

The level width $\Gamma$ in a kaonic atom is obtained from the eigenvalue $E_{K^{-}}-\mathrm{i} \Gamma / 2$ when solving the Klein-Gordon equation with an optical potential, $\left(E_{K^{-}}=m_{K^{-}}-B_{K^{-}}\right)$. It is also related to the imaginary part of the potential by the overlap integral of $\operatorname{Im} V_{K^{-}}$and $|\psi|^{2}$,

$$
\Gamma=-2 \frac{\int \operatorname{Im} V_{K^{-}}|\psi|^{2} d \vec{r}}{\int\left[1-\left(B_{K^{-}}+V_{\mathrm{C}}\right) / \mu_{K}\right]|\psi|^{2} d \vec{r}}
$$

where $B_{K^{-}}, V_{\mathrm{C}}$ and $\mu_{K}$ are the $K^{-}$binding energy, Coulomb potential and reduced mass, respectively, and $\psi$ is the $K^{-}$atomic wave function of the particular state concerned. When the best fit optical potential is $V_{K^{-}}^{(1)}+V_{K^{-}}^{(2)}$, the sum of a single-nucleon part and a multinucleon part, it is straight forward to calculate the fraction of single-nucleon absorptions, separately for any nucleus and for any specific kaonic atom state [19]. This fraction is calculated for each of the two levels involved in studies of strong interaction through the relative yield of 
the transition from an upper to a lower level and the energy shift and width of the lower level. From [21-23] we adopt $75 \pm 5 \%$ as an experimental result for this fraction.

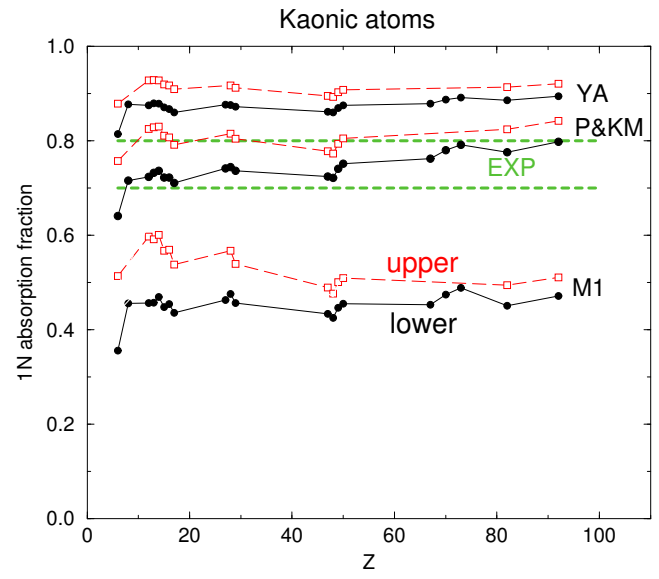

Figure 2. Calculated fractions of single-nucleon absorption for amplitudes M1, P, KM and YA augmented by the respective phenomenological term from Table 2. The $\mathrm{P}$ and $\mathrm{KM}$ results are indistinguishable on this figure. Solid circles and solid curves are for lower levels, open squares and long-dashed curves are for upper levels. Horizontal dashed lines mark the 70-80\% range of experimental values as discussed above.

Fig. 2 shows calculated single-nucleon absorption fractions at rest for kaonic atoms from carbon upwards based on results of Table 2. Experiments do not distinguish between absorptions from the upper and from the lower levels but from measured upper-level yields one can estimate that an average between the two corresponding curves is a good representative. Only for carbon (lowest Z) where the yield is exceptionally low the upper level results are the relevant ones. It is seen that the $\mathrm{P}$ and the $\mathrm{KM}$ amplitudes-based potentials agree well with experiment. The other three models from Fig. 1, not shown here, lead to too small fractions, similar to the M1 results. Therefore only the P and the KM amplitudes with their associated multinucleon phenomenological terms are able to describe both pionic atoms and single-nucleon absorption fractions.
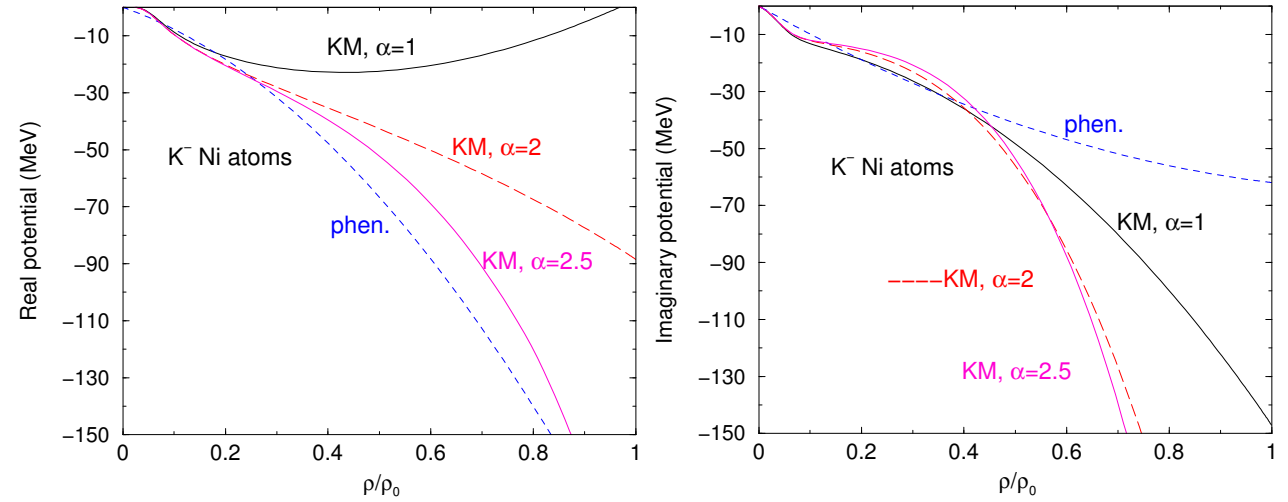

Figure 3. Best-fit $K^{-}$optical potentials for kaonic atoms of Ni. Solid curves and long-dashed curves are based on the KM single-nucleon amplitudes plus a phenomenological term $B\left(\rho / \rho_{0}\right)^{\alpha}$, dashed curves are for a purely phenomenological density-dependent best-fit potential [24]. All four potentials lead to equally good fits to the 65 data points for kaonic atoms.

It is interesting to return at this point to the best-fit optical potentials. Fig. 3 shows the KM amplitudes-based potentials including the respective multinucleon phenomenological 
parts. Potentials are ploted for three values of $\alpha$, all yielding equivalent fits to the data. Also shown is a purely phenomenological DD potential [24]. It is seen that the real potential is not determined for densities above $25 \%$ of the central value, but the imaginary potential is well-determined up to $50 \%$ of the central density.

\section{Summary}

Good global fits to kaonic atom data are possible with mixed chiral (single nucleon) and phenomenological multinucleon amplitudes within sub-threshold kinematics. Fractions of single-nucleon absorption favour only the $\mathrm{P}$ and the KM models. The real parts of optical potentials are not known above $25 \%$ of central density, thus it is impossible to answer the question of 'deep or shallow?' The imaginary parts of the potentials are known up to $50 \%$ of central density. That could constrain theories of multinucleon absorption.

Contributions of my colleagues in the Jerusalem-Prague collaboration, A. Cieplý, A. Gal, D. Gazda and $\mathbf{J}$. Mareš are gratefully acknowledged.

\section{References}

[1] C. J. Batty, Nucl. Phys. A 372418 (1981)

[2] E. Friedman, A. Gal, C. J. Batty, Nucl. phys. A 579518 (1994)

[3] A. Ramos, E. Oset, Nucl. Phys. A 671481 (2000)

[4] A Baca et al., Nucl. Phys. A 673335 (2000)

[5] A. Cieplý et al., Nucl. Phys. A 696 (2001)

[6] S. Wycech, Nucl. Phys. B 28541 (1971)

[7] W. A. Bardeen et al., Phys. Lett. 38B 135 (1972)

[8] M. Bazzi, et al., Phys. Lett. B 704113 (2011)

[9] M. Bazzi, et al., Nucl. Phys. A 88188 (2012)

[10] A. Cieplý, M. Mai, U.-G. Meißner, J. Smejkal, Nucl. Phys. A 95417 (2016)

[11] Y. Ikeda, T. Hyodo, W. Weise, Nucl. Phys. A 88198 (2012)

[12] A. Cieplý, J. Smejkal, Nucl. Phys. A 881115 (2012)

[13] Z.H. Guo, J.A. Oller, Phys. Rev. C 87035202 (2013)

[14] M. Mai, U.-G. Meißner, Eur. Phys. J. A 5130 (2015)

[15] T. Yamazaki, Y. Akaishi, Phys. Rev. C 76045201 (2007)

[16] A. Cieplý et al., Phys. Lett. B 702402 (2011)

[17] A. Cieplý et al., Phys. Rev. C 84045206 (2011)

[18] E. Friedman, A. Gal Nucl. Phys. A 89960 (2013)

[19] E. Friedman, A. Gal Nucl. Phys. A 95966 (2017)

[20] T. Waas, M. Rho, W. Weise, Nucl. Phys. A 617449 (1997)

[21] H. Davis et al., Nuovo Cimento 53313 (1968)

[22] J.W. Moulder et al., Nucl. Phys. B 35332 (1971)

[23] C. Vander Velde-Wilquet et al., Nuovo Cimento 39A 538 (1977)

[24] E. Friedman, A. Gal, Phys. Rep. 45289 (2007) 